\title{
The Changing Profile of Full-Time Faculty at Canadian Universities
}

\author{
MAX VON ZUR-MUEHLEN*
}

\begin{abstract}
Canadian universities underwent a remarkable expansion from the late sixties until the mid-seventies. However, they are entering the eighties on an uncertain note, due to financial restraints imposed by governments, the sudden growth in university enrolment and the shift to professionally oriented programs. These developments have had an impact on the socio-economic characteristics of the 33,000 full-time university teachers: their age, sex, academic rank, salary, citizenship, and qualifications. Especially uncertain is the demand for new faculty in this decade and the implications for the health of Canadian universities. This statistical series documents the changes which are occuring in the demand and supply patterns of doctoral recipients from Canadian universities with the purpose of providing a statistical base from which policy analyses could be developed.
\end{abstract}

\section{RÉSUMÉ}

Les universités canadiennes ont subi au cours des années 1960/mi-1970 un épanouissement remarquable. Cependant, elles se voient entrer dans une période d'incertitude au début des années 1980 due aux contraintes financières imposées par les gouvernements, l'accroissement soudain de l'inscription au niveau universitaire, et au déplacement vers les programmes de métier. Ces développements ont eu un impact sur les caractéristiques socio-économiques des quelques 33,000 professeurs engagés à plein temps soit l'âge, le sexe, le rang académique, le salaire, la citoyenneté et les qualifications. Comme la demande de professeurs est restreinte dans cette décade, le fonctionnement des universités canadiennes en est affecté. Les différentes statistiques présentent les changements qui surviennent face aux modèles d'offre et de demande chez les détenteurs de doctorats d'universités canadiennes. Cette documentation a pour but de fournir une ligne de conduite qui servira au développement de nouvelles politiques.

*Co-Ordinator of Research: Education, Science and Culture Division, Statistics Canada. 


\section{INTRODUCTION}

The purpose of the documentation is to provide a statistical overview of full-time faculty at Canadian universities. The data have been derived from the Statistics Canada University Teachers file. No attempt is made to assess the implications of the tables, but they do constitute a base from which such analyses could be developed.

During the last 15 years the demographic and socio-economic characteristics of full-time university teachers have changed substantially. These trends assume added significance because of the severe financial restraints imposed by governments and the sudden growth in university enrolment during the early eighties. In the two years, 1981.82 and 1982-83, full-time enrolment grew by more than 10 percent, and the shift in student preferences to professionally oriented programs continued.

This article traces, within an historical context, changes in the age, sex, academic rank, salary and citizenship of full-time teachers at Canadian universities.

At the same time, an attempt is made to estimate, for this decade, both the replacement demand for full-time university teachers and the supply of doctoral degrees available from Canadian universities.

\section{FULL-TIME UNIVERSITY TEACHERS}

The number of full-time university teachers increased sixfold from 5,000 during the mid-fifties to about 30,000 by $1974-75$. Since then this number has levelled off at about 33,000.

A basic assumption of this article is that despite shifts in the distribution of full-time faculty from arts and science to professionally oriented disciplines, the total number of teachers will not decline drastically during the next few years.

This picture differs from the late sixties and early seventies when, for about ten years, an average of about 2,000 additional full-time teachers were added to the expanding Canadian university system each year.

University teaching has long been the preserve of males, and this pattern continues only slightly changed into the 1980 s. In $1958-59$ only $11 \%$ of university teachers were women, a proportion that increased to $13 \%$ in 1970.71 and to $15 \%$ in 1980-81 (Table 1). In education and fine and applied arts, the proportion of full-time female faculty has been above $20 \%$. Over the years, the percentage of women teaching engineering, and mathematics and physical sciences has remained small ( $1 \%$ and $5 \%$, respectively).

Without special efforts or incentives to hire them, women may continue to be underrepresented because of the expected lack of employment opportunities at Canadian universities for the next fifteen years.

\section{AGE STRUCTURE}

This scarcity of positions for new faculty affects another important variable the age structure of full-time university teachers. In $1974-75,69 \%$ of them were 
TABLE 1. Full-time University Teachers by Teaching Field and Sex, 1980-81

\begin{tabular}{|c|c|c|c|}
\hline Teaching Field & $\begin{array}{r}\text { Male } \\
(\%)\end{array}$ & $\begin{array}{c}\text { Female } \\
(\%)\end{array}$ & $\begin{array}{c}\text { Total } \\
\text { (No. and \%) }\end{array}$ \\
\hline Education & $\begin{array}{c}76 \\
(80)\end{array}$ & $\begin{array}{c}24 \\
(20)\end{array}$ & $\begin{array}{r}3,148 \\
10.0\end{array}$ \\
\hline Fine and Applied Arts & $\begin{array}{c}79 \\
(85)\end{array}$ & $\begin{array}{c}21 \\
(15)\end{array}$ & $\begin{array}{r}1,382 \\
4.4\end{array}$ \\
\hline Humanities & $\begin{array}{c}82 \\
(83)\end{array}$ & $\begin{array}{c}18 \\
(17)\end{array}$ & $\begin{array}{r}5,549 \\
17.7\end{array}$ \\
\hline Social Sciences & $\begin{array}{l}86 \\
(91)\end{array}$ & $\begin{array}{l}14 \\
(9)\end{array}$ & $\begin{array}{r}8,237 \\
26.3\end{array}$ \\
\hline $\begin{array}{l}\text { Agriculture and Biological } \\
\text { Sciences }\end{array}$ & $\begin{array}{c}83 \\
(84)\end{array}$ & $\begin{array}{c}17 \\
(16)\end{array}$ & $\begin{array}{r}1,983 \\
6.3\end{array}$ \\
\hline Engineering \& Applied Sciences & $\begin{array}{c}99 \\
(99)\end{array}$ & $\stackrel{1}{(1)}$ & $\begin{array}{r}2,395 \\
7.6\end{array}$ \\
\hline Health Sciences & $\begin{array}{c}78 \\
(79)\end{array}$ & $\begin{array}{c}22 \\
(21)\end{array}$ & $\begin{array}{r}4,523 \\
14.4\end{array}$ \\
\hline Mathematics \& Physical Sciences & $\begin{array}{c}95 \\
(96)\end{array}$ & $\begin{array}{c}5 \\
(4)\end{array}$ & $\begin{array}{r}4,165 \\
13.3\end{array}$ \\
\hline TOTAL & $\begin{array}{c}85 \\
(87)\end{array}$ & $\begin{array}{c}15 \\
(13)\end{array}$ & $\begin{array}{c}31,382 \star \\
100.0\end{array}$ \\
\hline
\end{tabular}

*Excludes 1,917 unclassified and not reported full-time university teachers.

Note: Percentages in brackets show the sex distribution for 1970-7l.

35 or older (Table 2). By 1982-83 the proportion had risen to $86 \%$. Meanwhile, the under 30 group dropped from $8 \%$ of the total to $3 \%$. The accompanying rise in the median age since the mid-seventies from 39 to 44 indicates the same pattern.

The age structure of full-time university teachers varies among disciplines, reflecting the stage of development and demand for each one. In 1980, more than $20 \%$ of the teachers in disciplines such as agriculture, forestry, dentistry, and library science were older than 55 . In contrast, fewer than $10 \%$ in linguistics, business, law, and psychology were 55 or older. 
TABLE 2. Age Distribution of Full-time University Teachers, 1971-72 to 1982-83

\begin{tabular}{|c|c|c|c|c|c|c|c|c|c|c|}
\hline Age Group & $1971-72$ & $1974-75$ & $1975-76$ & $1976-77$ & $1977-78$ & $1978-79$ & $1979-80$ & $1980-81$ & $1981 \rightarrow 82 \star$ & $1982-83^{*}$ \\
\hline $20-24$ & 0.9 & 0.4 & 0.4 & 0.4 & 0.3 & 0.2 & 0.2 & 0.4 & 0.2 & 0.2 \\
\hline $25-29$ & 13.8 & 7.7 & 6.9 & 5.8 & 5.2 & 4.5 & 3.9 & 3.5 & 3.1 & 2.9 \\
\hline $30-34$ & 23.6 & 22.7 & 21.4 & 19.9 & 18.0 & 15.7 & 14.1 & 12.6 & 11.0 & 10.1 \\
\hline SUB-TOTAL $(20-34)$ & 38.3 & 30.8 & 28.7 & 26.1 & 23.5 & 20.4 & 18.2 & 16.5 & 14.3 & 13.2 \\
\hline $35-39$ & 20.2 & 21.9 & 22.3 & 22.6 & 23.4 & 23.7 & 23.3 & 22.1 & 20.2 & 18.6 \\
\hline $40-44$ & 15.6 & 17.3 & 17.5 & 18.1 & 18.3 & 19.2 & 19.9 & 20.8 & 21.5 & 22.0 \\
\hline $45-49$ & 11.2 & 12.6 & 13.2 & 13.8 & 14.4 & 14.9 & 15.5 & 16.1 & 16.8 & 17.6 \\
\hline SUB-TOTAL $(35-39)$ & 47.0 & 51.8 & 53.0 & 54.5 & 56.1 & 57.8 & 58.7 & 59.0 & 58.5 & 58.2 \\
\hline $50-54$ & 7.3 & 9.0 & 9.3 & 9.7 & 10.0 & 10.7 & 11.3 & 11.9 & 12.7 & 13.8 \\
\hline $55-59$ & 4.6 & 5.2 & 5.7 & 6.2 & 6.6 & 7.1 & 7.7 & 8.3 & 9.2 & 9.2 \\
\hline $60-64$ & 2.8 & 3.2 & 3.3 & 3.5 & 3.8 & 4.0 & 4.1 & 4.3 & 5.3 & 5.6 \\
\hline SUB-TOTAL $(50-64)$ & 14.7 & 17.4 & 18.3 & 19.4 & 20.4 & 21.8 & 23.1 & 24.5 & 27.2 & 28.6 \\
\hline TOTAL (percent) & 100.0 & 100.0 & 100.0 & 100.0 & 100.0 & 100.0 & 100.0 & 100.0 & 100.0 & 100.0 \\
\hline TOTAL NUMBER REPORTED & 26,675 & 29,672 & 30,498 & 31,280 & 31,895 & 32,357 & 32,500 & 31,107 & 25,713 & 28,196 \\
\hline Not reported & 99 & 75 & 61 & 138 & 37 & 48 & 58 & 1,917 & - & - \\
\hline older than 64 & 200 & 212 & 225 & 230 & 235 & 240 & 245 & 275 & 177 & 114 \\
\hline GRAND TOTAL & 26,973 & 29,959 & 30,784 & 31,648 & 32,167 & 32,645 & 32,803 & 33,299 & 25,890 & 18,310 \\
\hline Median Age & 38 & 39 & 39 & 40 & 40 & 41 & 42 & 42 & 43 & 44 \\
\hline
\end{tabular}

*Excludes Quebec Institutions, and for 1982-83, some other universities. 
31 The Changing Profile of Full-Time Faculty at Canadian Universities

TABLE 3. Actual and Projected Academic Rank Distribution of full-time University Teachers, 1967-68 to 1990-91

\begin{tabular}{ccccc}
\hline $\begin{array}{c}\text { Academic } \\
\text { year }\end{array}$ & $\begin{array}{c}\text { Full } \\
\text { professor }\end{array}$ & $\begin{array}{c}\text { Associate } \\
\text { professor }\end{array}$ & $\begin{array}{c}\text { Sub-total Assistant Rank below Sub-total } \\
\text { two senior professor } \\
\text { ranks }\end{array}$ & $\begin{array}{c}\text { assistant two junior } \\
\text { professor ranks }\end{array}$ \\
\hline
\end{tabular}

(Yercent)

Actual:

$\begin{array}{lllllll}1967-68 & 18.5 & 25.2 & 43.7 & 37.0 & 19.3 & 56.3 \\ 1968-69 & 18.5 & 26.3 & 44.8 & 37.9 & 17.3 & 55.2 \\ 1969-70 & 18.6 & 26.8 & 45.4 & 38.0 & 16.6 & 54.6 \\ 1970-71 & 18.8 & 27.2 & 46.0 & 37.7 & 16.3 & 54.0 \\ 1971-72 & 21.3 & 29.1 & 50.4 & 37.4 & 12.2 & 49.6 \\ 1972-73 & 21.7 & 29.9 & 51.6 & 37.2 & 11.1 & 48.3 \\ 1973-74 & 23.2 & 32.5 & 55.7 & 34.7 & 9.8 & 44.5 \\ 1974-75 & 24.5 & 34.2 & 58.7 & 33.2 & \mathbf{8 . 1} & 41.3 \\ 1975-76 & 25.7 & 35.6 & 61.3 & 31.3 & 7.4 & 38.7 \\ 1976-77 & 26.7 & 37.1 & 63.8 & 29.9 & 6.5 & 36.4 \\ 1977-78 & 27.8 & 37.8 & 65.6 & 28.1 & 6.3 & 34.4 \\ 1978-79 & 28.7 & 39.3 & 68.0 & 26.2 & 5.8 & 32.0 \\ 1979-80 & 30.2 & 39.7 & 69.9 & 24.7 & 5.4 & 30.1 \\ 1980-81 & 31.2 & 40.0 & 71.2 & 23.4 & 5.4 & 28.8 \\ 1981-82 * & 33.5 & 39.2 & 72.7 & 22.2 & 5.1 & 27.3 \\ 1982-83^{*} & 34.8 & 38.6 & 73.4 & 21.1 & 5.5 & 26.6\end{array}$

Projected:

$\begin{array}{lllllll}1983-84 & 35.7 & 38.7 & 74.4 & 20.2 & 5.4 & 25.6 \\ 1984-85 & 36.6 & 38.8 & 75.4 & 19.3 & 5.3 & 24.6 \\ 1985-86 & 37.5 & 38.9 & 76.4 & 18.4 & 5.2 & 23.6 \\ 1986-87 & 38.4 & 39.0 & 77.4 & 17.5 & 5.1 & 22.6 \\ 1987-88 & 39.3 & 39.1 & 78.4 & 16.6 & 5.0 & 21.6 \\ 1988-89 & 40.2 & 39.2 & 79.4 & 15.7 & 4.9 & 20.6 \\ 1989-90 & 41.1 & 39.3 & 80.4 & 14.8 & 4.8 & 19.6 \\ 1990-91 & 42.0 & 39.4 & 81.4 & 13.9 & 4.7 & 18.6\end{array}$

*Preliminary

Note: It has been estimated that 0.9 percent of the university cohort will be promoted from associate to full professor, and 0.1 percent from assistant to associate professor. Under these assumptions, the two junior ranks decline proportionately. 


\section{ACADEMIC RANK DISTRIBUTION}

Academic rank distribution is related to teachers' age structure. In 1956-57, 52\% of the full-time teachers were at the senior ranks (full and associate professor). During the expansionary sixties, this percentage fell to a low of $44 \%$ in $1967-68$ (Table 3). It then rose gradually to $73 \%$ by $1982-83$. Conversely, the proportions in the two junior ranks (assistant professor and instructor/lecturer) declined from $56 \%$ of the total in $1967-68$, to $27 \%$ in $1982-83$. Of particular note is the decrease of the rank below assistant prpfessor from 19\% in 1967-68 to the present $6 \%$, further evidence of reduced hiring of junior faculty over the last fifteen years. If these trends continue, and if one assumes normal progression through the ranks as well as no net additions to full-time academic staff, the proportion at the two senior levels could rise to above $80 \%$ within a few years, even with a slower promotion rate.

\section{SALARY STRUCTURE}

The median salary of all full-time university teachers almost quadrupled between $1967-68$ and $1982-83$ from $\$ 11,400$ to $\$ 43,000$ (Table 4 ). The median for full professors went from $\$ 17,100$ to $\$ 54,900$; for assistant professors from $\$ 10,200$ to $\$ 32,000$. The financial implications of the rising percentage of teachers who have entered and will enter the senior ranks are obvious. To maintain current levels of remuneration, a larger share of university budgets will have to be allocated to teachers' salaries. But at the same time, demands from other expenditure sectors, such as non-academic salaries, administration, libraries, maintenance, research, and student assistance, are increasing.

An important factor in the higher education expenditure pattern is that university education is a labour-intensive service industry whose rate of inflation has been higher than that of the general economy. Growth of the median salary can be compared with the Consumer Price Index (CPI). Using 1967-68 as a base of 100.0, the median salary for all academic ranks increased to 377.3 in 1981-82, while the CPI rose to 303.6 in 1982 .

To counteract escalating costs, some universities have already started to reduce the number of full-time faculty and/or have decided not to replace those on sabbatical leave. Other institutions are substituting part-time for full-time teachers, at substantial savings. Nevertheless, the cost per student, in constant dollars, is likely to grow. Opportunities for adjustment vary by size of institution; small universities have less flexibility.

\section{FOREIGN FACULTY}

The proportion of newly appointed full-time faculty with Canadian citizenship increased from $59 \%$ in $1972-73$ to an estimated $75 \%$ in $1982-83$ (Table 5). However, for a variety of reasons, Canadian universities must in some instances continue to rely on foreign faculty. In certain disciplines for which demand is heavy, 


\begin{tabular}{|c|c|c|c|c|c|c|c|c|c|c|}
\hline Academic Rank & $1967-68$ & $1969-70$ & $1971-72$ & $1973-74$ & $1975-76$ & $1977-78$ & $1979-80$ & $1980-81$ & $1981-82 *$ & $1982-83^{*}$ \\
\hline & \multicolumn{10}{|c|}{ (Current dollars) } \\
\hline Full professor & 17,100 & 19,900 & 22,600 & 25,200 & 31,500 & 36,500 & 41,500 & 45,300 & 49,600 & 54,900 \\
\hline Associate professor & 13,000 & 14,000 & 26,800 & 18,600 & 23,100 & 27,700 & 31,700 & 34,700 & 37,600 & 41,700 \\
\hline Assistant professor & 10,200 & 11,800 & 13,300 & 14,700 & 18,600 & 22,000 & 25,000 & 27,400 & 29,300 & 32,000 \\
\hline Rank below assistant professor & 8,000 & 9,400 & 10,500 & 11,800 & 14,900 & 17,800 & 20,200 & 21,900 & 23,600 & 26,000 \\
\hline TOTAL & 11,400 & 13,300 & 15,100 & 17,200 & 22,400 & 27,300 & 31,800 & 35,200 & 38,700 & 43,000 \\
\hline Salary Index (total) & 100.0 & 116.3 & 132.3 & 150.4 & 196.0 & 239.0 & 278.9 & 309.0 & 339.1 & 377.3 \\
\hline $\begin{array}{l}\text { Consumer Price Index } \\
\text { (Calendar year) }\end{array}$ & 100.0 & 108.8 & 115.6 & 130.2 & 160.1 & 185.9 & 221.0 & 243.5 & 274.0 & 303.6 \\
\hline $\begin{array}{l}\text { U.S. Higher Education } \\
\text { Price Index }\end{array}$ & 100.0 & 113.2 & 128.6 & 143.0 & 166.1 & 188.7 & 216.9 & 238.3 & $\mathbf{N} / \mathbf{A}$ & N/A \\
\hline
\end{tabular}


TABLE 5. Citizenshtp of Newly Appointed Full-time University Teachers, 1972-73 to 1982-83

\begin{tabular}{|c|c|c|c|c|c|c|c|c|c|c|}
\hline $\begin{array}{c}\text { Year of } \\
\text { Appointment }\end{array}$ & Canada & $\begin{array}{l}\text { United } \\
\text { States }\end{array}$ & $\begin{array}{l}\text { Un1ted } \\
\text { KIngdom }\end{array}$ & $\begin{array}{c}\text { Other } \\
\text { Commonwealth }\end{array}$ & $\begin{array}{c}\text { France and } \\
\text { Belg1 um }\end{array}$ & $\begin{array}{l}\text { Other } \\
\text { Europe }\end{array}$ & $\begin{array}{c}\text { Other } \\
\text { Countries }\end{array}$ & Sub-total & $\begin{array}{l}\text { Not } \\
\text { reported }\end{array}$ & Total \\
\hline $1972-73$ & $\begin{array}{r}1,094 \\
59.1\end{array}$ & $\begin{array}{r}332 \\
17.9\end{array}$ & $\begin{array}{l}165 \\
8.9\end{array}$ & $\begin{array}{r}69 \\
3.7\end{array}$ & $\begin{array}{r}56 \\
3.0\end{array}$ & $\begin{array}{r}66 \\
3.6\end{array}$ & $\begin{array}{r}68 \\
3.7\end{array}$ & $\begin{array}{l}1,850 \\
100.0\end{array}$ & 456 & 2,306 \\
\hline $1973-74$ & $\begin{array}{r}1,108 \\
63.3\end{array}$ & $\begin{array}{r}296 \\
16.9\end{array}$ & $\begin{array}{l}134 \\
7.7\end{array}$ & $\begin{array}{r}63 \\
3.6\end{array}$ & $\begin{array}{r}31 \\
1.8\end{array}$ & $\begin{array}{r}67 \\
3.8\end{array}$ & $\begin{array}{r}51 \\
2.9\end{array}$ & $\begin{array}{l}1,750 \\
100.0\end{array}$ & 187 & 1,937 \\
\hline $1974-75$ & $\begin{array}{r}1,280 \\
62.4\end{array}$ & $\begin{array}{r}361 \\
17.6\end{array}$ & $\begin{array}{l}177 \\
8.6\end{array}$ & $\begin{array}{r}64 \\
3.1\end{array}$ & $\begin{array}{r}43 \\
2.1\end{array}$ & $\begin{array}{r}57 \\
2.8\end{array}$ & $\begin{array}{r}69 \\
3.4\end{array}$ & $\begin{array}{l}2,051 \\
100.0\end{array}$ & 203 & 2,254 \\
\hline $1975-76$ & $\begin{array}{r}1,293 \\
64.2\end{array}$ & $\begin{array}{r}325 \\
16.1\end{array}$ & $\begin{array}{l}144 \\
7.1\end{array}$ & $\begin{array}{r}78 \\
3.9\end{array}$ & $\begin{array}{r}40 \\
2.0\end{array}$ & $\begin{array}{r}62 \\
3.1\end{array}$ & $\begin{array}{r}72 \\
3.6\end{array}$ & $\begin{array}{l}2,014 \\
100.0\end{array}$ & 134 & 2,148 \\
\hline $1976-77$ & $\begin{array}{r}1,399 \\
65.3\end{array}$ & $\begin{array}{r}319 \\
14.9\end{array}$ & $\begin{array}{l}154 \\
7.2\end{array}$ & $\begin{array}{r}87 \\
4.0\end{array}$ & $\begin{array}{r}44 \\
2.0\end{array}$ & $\begin{array}{r}57 \\
2.7\end{array}$ & $\begin{array}{r}84 \\
3.9\end{array}$ & $\begin{array}{l}2,144 \\
100.0\end{array}$ & 18 & 2,162 \\
\hline $1977-78$ & $\begin{array}{r}1,422 \\
69.2\end{array}$ & $\begin{array}{r}273 \\
13.3\end{array}$ & $\begin{array}{l}148 \\
7.2\end{array}$ & $\begin{array}{r}56 \\
2.7\end{array}$ & $\begin{array}{r}41 \\
2.0\end{array}$ & $\begin{array}{r}48 \\
2.3\end{array}$ & $\begin{array}{r}67 \\
3.3\end{array}$ & $\begin{array}{l}2,055 \\
100.0\end{array}$ & 51 & 2,106 \\
\hline $1978-79$ & $\begin{array}{r}1,743 \\
73.7\end{array}$ & $\begin{array}{r}250 \\
10.6\end{array}$ & $\begin{array}{l}153 \\
6.5\end{array}$ & $\begin{array}{r}63 \\
2.7\end{array}$ & $\begin{array}{r}37 \\
1.5\end{array}$ & $\begin{array}{r}56 \\
2.4\end{array}$ & $\begin{array}{r}62 \\
2.6\end{array}$ & $\begin{array}{l}2,364 \\
100.0\end{array}$ & 36 & 2,400 \\
\hline $1979-80$ & $\begin{array}{r}1,380 \\
72.0\end{array}$ & $\begin{array}{r}209 \\
10.9\end{array}$ & $\begin{array}{l}134 \\
7.0\end{array}$ & $\begin{array}{r}64 \\
3.4\end{array}$ & $\begin{array}{r}22 \\
1.1\end{array}$ & $\begin{array}{r}49 \\
2.6\end{array}$ & $\begin{array}{r}58 \\
3.0\end{array}$ & $\begin{array}{l}1,916 \\
100.0\end{array}$ & 53 & 1,969 \\
\hline $1980-81^{*}$ & $\begin{array}{r}1,301 \\
72.2\end{array}$ & $\begin{array}{r}234 \\
13.0\end{array}$ & $\begin{array}{l}102 \\
5.7\end{array}$ & $\begin{array}{r}51 \\
2.8\end{array}$ & $\begin{array}{r}9 \\
0.5\end{array}$ & $\begin{array}{r}37 \\
2.1\end{array}$ & $\begin{array}{r}66 \\
3.7\end{array}$ & $\begin{array}{l}1,800 \\
100.0\end{array}$ & 63 & 1,863 \\
\hline $1981-82^{*}$ & $\begin{array}{r}1,301 \\
72.2\end{array}$ & $\begin{array}{r}206 \\
11.4\end{array}$ & $\begin{array}{l}104 \\
5.8\end{array}$ & $\begin{array}{r}64 \\
3.6\end{array}$ & $\begin{array}{r}14 \\
0.8\end{array}$ & $\begin{array}{r}46 \\
2.6\end{array}$ & $\begin{array}{r}65 \\
3.6\end{array}$ & $\begin{array}{l}1,800 \\
100.0\end{array}$ & 92 & 1,892 \\
\hline $1982-83^{\star}$ & $\begin{array}{r}961 \\
75.2\end{array}$ & $\begin{array}{l}109 \\
8.5\end{array}$ & $\begin{array}{r}64 \\
5.0\end{array}$ & $\begin{array}{r}45 \\
3.5\end{array}$ & $\begin{array}{r}13 \\
1.0\end{array}$ & $\begin{array}{r}36 \\
2.8\end{array}$ & $\begin{array}{r}50 \\
3.9\end{array}$ & $\begin{array}{l}1,278 \\
100.0\end{array}$ & 44 & 1,322 \\
\hline 11-year total & $\begin{array}{r}14,282 \\
67.9\end{array}$ & $\begin{array}{r}2,914 \\
13.9\end{array}$ & $\begin{array}{r}1,479 \\
7.0\end{array}$ & $\begin{array}{l}704 \\
3.3\end{array}$ & $\begin{array}{l}350 \\
1.7\end{array}$ & $\begin{array}{l}581 \\
2.8\end{array}$ & $\begin{array}{l}712 \\
3.4\end{array}$ & $\begin{array}{r}21,022 \\
100.0\end{array}$ & 1,337 & 22,359 \\
\hline Total faculty $1980-81$ & $\begin{array}{r}23,943 \\
76.4\end{array}$ & $\begin{array}{r}3,743 \\
11.9\end{array}$ & $\begin{array}{r}1,406 \\
4.5\end{array}$ & $\begin{array}{l}596 \\
1.9\end{array}$ & $\begin{array}{l}389 \\
1.2\end{array}$ & $\begin{array}{l}555 \\
1.8\end{array}$ & $\begin{array}{l}459 \\
1.5\end{array}$ & $\begin{array}{r}31,091 \\
100.0\end{array}$ & 291 & 31,382 \\
\hline
\end{tabular}

*Excludes Quebec institutions, and for 1982-83 some other universities. 
such as management and administrative studies, Canada produces an insufficient number of Ph.D.'s. Moreover, not enough senior persons with strong research experience are available in Canada. In addition, universities are by nature internationally oriented. Therefore, Canadian universities will continue to employ foreign faculty, although to a decreasing rate. The extent to which this has been true in the past is shown in Table 6.

Although it is not infallible, the geographic origin of the teachers' first degree can serve as an indicator of their nationality.

Overall, in 1980-81, 58\% had obtained their first degree in Canada, including those who might have been permanent residents (landed immigrants). The second largest group, 17\%, had graduated in the United States, and 10\% had earned their first degree in the United Kingdom. In absolute numbers, 12,874 of the 30,861 full-time faculty had obtained their first degree abroad. However, variations among the eight fields were substantial: from a low of $30 \%$ in education to a high of $54 \%$ in fine and applied arts. Compared with the social sciences and humanities, a disproportionately large number in the natural and physical sciences had earned their first degree in countries other than Canada, the United States or the United Kingdom.

At the discipline level, in some social sciences such as archaeology and anthropology, American degrees outnumbered Canadian. This contrasts with most of the applied disciplines, in which better than two-thirds of the teachers had graduated from Canadian universities.

\section{DEMAND AND SUPPLY OF FACULTY}

An obvious consequence of the age structure (72\% are between 35 and 54 ) is that few university teaching jobs will open up in the near future. During the period 1983 to 1991 , just $13 \%$ of full-time teachers will reach the normal retirement age of 65 (Table 7). This means that retirement will free about 4,200 fulltime positions. An estimated 1,500 more will be available on account of mortality. Consequently, retirement and mortality will create around 5,700 openings: an average of about 634 a year. But this may be an overestimation. Elimination of the mandatory retirement age in provinces such as New Brunswick, Quebec and Manitoba has reduced the retirement rate substantially, partly because of the unfavourable economic climate which erodes pensions. Moreover, possible financial savings and anticipation of an absolute decline in full-time enrolment might cause universities to leave these positions vacant, to replace them with temporary (term) appointments or to make greater use of part-time teachers. Such measures would further restrict employment opportunities. Besides retirement and mortality rates, a third variable affecting the need for replacement is mobility. Although no systematic attempt has been made in Canada to find out what happens to faculty who resign, there is evidence that mobility between universities and other employment sectors (and vice versa) is declining, not a surprising phenomenon in a strained labour market. Therefore, zero net mobility has been assumed. 
TABLE 6. Geographic Area of First Degree of Full-time University Teachers by Teaching Field, 1980-81

\begin{tabular}{|c|c|c|c|c|c|c|c|c|}
\hline & Canada & $\begin{array}{l}\text { United } \\
\text { States }\end{array}$ & $\begin{array}{l}\text { United } \\
\text { Kingdom }\end{array}$ & $\begin{array}{l}\text { Other } \\
\text { Common- } \\
\text { wealth }\end{array}$ & $\begin{array}{l}\text { France } \\
\text { and } \\
\text { Belgium }\end{array}$ & $\begin{array}{l}\text { Other } \\
\text { Europe }\end{array}$ & other & Total \\
\hline Education & $\begin{array}{l}2,179 \\
(70.2)\end{array}$ & $\begin{array}{c}551 \\
(17.7)\end{array}$ & $\begin{array}{c}160 \\
(5.1)\end{array}$ & $\begin{array}{c}67 \\
(2.2)\end{array}$ & $\begin{array}{c}50 \\
(1.6)\end{array}$ & $\begin{array}{c}49 \\
(1.6)\end{array}$ & $\begin{array}{c}49 \\
(1.6)\end{array}$ & $\begin{array}{c}3,105 \\
(100.0)\end{array}$ \\
\hline Fine and Applied Arts & $\begin{array}{c}555 \\
(45.7)\end{array}$ & $\begin{array}{c}440 \\
(36.3)\end{array}$ & $\begin{array}{c}105 \\
(8.7)\end{array}$ & $\begin{array}{c}11 \\
(0.9)\end{array}$ & $\begin{array}{c}20 \\
(1.6)\end{array}$ & $\begin{array}{c}59 \\
(4.9)\end{array}$ & $\begin{array}{c}23 \\
(1.9)\end{array}$ & $\begin{array}{c}1,213 \\
(100.0)\end{array}$ \\
\hline Humanities & $\begin{array}{l}2,903 \\
(52.9)\end{array}$ & $\begin{array}{l}1,236 \\
(22.5)\end{array}$ & $\begin{array}{c}629 \\
(11.5)\end{array}$ & $\begin{array}{c}76 \\
(1.4)\end{array}$ & $\begin{array}{c}226 \\
(4.1)\end{array}$ & $\begin{array}{c}290 \\
(5.3)\end{array}$ & $\begin{array}{c}123 \\
(2.3)\end{array}$ & $\begin{array}{c}5,483 \\
(100.0)\end{array}$ \\
\hline Soctal Sclences & $\begin{array}{l}4,673 \\
(57.3)\end{array}$ & $\begin{array}{l}1,764 \\
(21.6)\end{array}$ & $\begin{array}{c}633 \\
(7.7)\end{array}$ & $\begin{array}{c}293 \\
(3.6)\end{array}$ & $\begin{array}{c}251 \\
(3.1)\end{array}$ & $\begin{array}{l}240 \\
(2.9)\end{array}$ & $\begin{array}{c}307 \\
(3.8)\end{array}$ & $\begin{array}{c}8,161 \\
(100.0)\end{array}$ \\
\hline SUB-TOTAL - HUMAN SCIENCES & $\begin{array}{c}10,310 \\
(57.4)\end{array}$ & $\begin{array}{l}3,991 \\
(22.2)\end{array}$ & $\begin{array}{c}1,527 \\
(8.5)\end{array}$ & $\begin{array}{c}447 \\
(2.5)\end{array}$ & $\begin{array}{c}547 \\
(3.0)\end{array}$ & $\begin{array}{c}638 \\
(3.6)\end{array}$ & $\begin{array}{c}502 \\
(2.8)\end{array}$ & $\begin{array}{l}17,962 \\
(100.0)\end{array}$ \\
\hline Agriculture \& Biological Sciences & $\begin{array}{l}1,155 \\
(58.6)\end{array}$ & $\begin{array}{c}331 \\
(16.8)\end{array}$ & $\begin{array}{c}243 \\
(12.3)\end{array}$ & $\begin{array}{c}92 \\
(4.7)\end{array}$ & $\begin{array}{c}21 \\
(1.1)\end{array}$ & $\begin{array}{c}69 \\
(3.5)\end{array}$ & $\begin{array}{c}59 \\
(3.0)\end{array}$ & $\begin{array}{c}1,970 \\
(100.0)\end{array}$ \\
\hline Engineering and Applied Sciences & $\begin{array}{l}1,373 \\
(57.9)\end{array}$ & $\begin{array}{c}159 \\
(6.7)\end{array}$ & $\begin{array}{c}296 \\
(12.5)\end{array}$ & $\begin{array}{c}134 \\
(5.7)\end{array}$ & $\begin{array}{c}64 \\
(2.7)\end{array}$ & $\begin{array}{c}170 \\
(7.2)\end{array}$ & $\begin{array}{l}174 \\
(7.3)\end{array}$ & $\begin{array}{c}2,370 \\
(100.0)\end{array}$ \\
\hline Health Sciences & $\begin{array}{l}2,971 \\
(66.9)\end{array}$ & $\begin{array}{c}302 \\
(6.8)\end{array}$ & $\begin{array}{c}539 \\
(12.1)\end{array}$ & $\begin{array}{c}173 \\
(3.9)\end{array}$ & $\begin{array}{c}46 \\
(1.0)\end{array}$ & $\begin{array}{c}211 \\
(4.8)\end{array}$ & $\begin{array}{c}200 \\
(4.5)\end{array}$ & $\begin{array}{c}4,442 \\
(100.0)\end{array}$ \\
\hline Mathematics and Physical Sciences & $\begin{array}{l}2,178 \\
(52.9)\end{array}$ & $\begin{array}{c}516 \\
(12.5)\end{array}$ & $\begin{array}{c}602 \\
(14.6)\end{array}$ & $\begin{array}{c}278 \\
(6.8)\end{array}$ & $\begin{array}{c}84 \\
(2.1)\end{array}$ & $\begin{array}{c}256 \\
(6.2)\end{array}$ & $\begin{array}{c}203 \\
(4.9)\end{array}$ & $\begin{array}{c}4,117 \\
(100.0)\end{array}$ \\
\hline SUB-TOTAL - SCIENCES & $\begin{array}{l}7,677 \\
(59.5)\end{array}$ & $\begin{array}{l}1,308 \\
(10.1)\end{array}$ & $\begin{array}{l}1,680 \\
(13.0)\end{array}$ & $\begin{array}{c}677 \\
(5.3)\end{array}$ & $\begin{array}{c}215 \\
(1.7)\end{array}$ & $\begin{array}{c}706 \\
(5.5)\end{array}$ & $\begin{array}{c}636 \\
(4.9)\end{array}$ & $\begin{array}{l}12,899 \\
(100.0)\end{array}$ \\
\hline TOTAL & $\begin{array}{r}17,987 \\
(58.3)\end{array}$ & $\begin{array}{l}5,299 \\
(17.2)\end{array}$ & $\begin{array}{l}3,207 \\
(10.4)\end{array}$ & $\begin{array}{r}1,124 \\
(3.6)\end{array}$ & $\begin{array}{c}762 \\
(2.5)\end{array}$ & $\begin{array}{r}1,344 \\
(4.3)\end{array}$ & $\begin{array}{c}1,138 \\
(3.7)\end{array}$ & $\begin{array}{l}30,861 \\
(100.0)\end{array}$ \\
\hline
\end{tabular}


37 The Changing Profile of Full-Time Faculty at Canadian Universities

TABLE 7. Projected Replacement Positions Available for Full-time University Teachers, 1983 to 1991

\begin{tabular}{|c|c|c|c|c|c|c|}
\hline & $\begin{array}{l}\text { Retire- } \\
\text { ments } \\
\text { (No.) }\end{array}$ & $\begin{array}{c}\text { Retirement } \\
\text { rate } \\
(\%)\end{array}$ & $\begin{array}{c}\text { Mortality } \\
(\%)\end{array}$ & $\begin{array}{l}\text { Mortality } \\
\text { rate } \\
(\%)\end{array}$ & $\begin{array}{l}\text { Total } \\
\text { replacement } \\
\text { (No.) }\end{array}$ & $\begin{array}{l}\text { Replacement } \\
\text { rate } \\
(\%)\end{array}$ \\
\hline 1983 & 265 & 0.8 & 165 & 0.5 & 430 & 1.3 \\
\hline 1984 & 309 & 0.9 & 165 & 0.5 & 474 & 1.4 \\
\hline 1985 & 388 & 1.2 & 165 & 0.5 & 553 & 1.7 \\
\hline 1986 & 445 & 1.4 & 165 & 0.5 & 610 & 1.9 \\
\hline 1987 & 474 & 1.4 & 165 & 0.5 & 639 & 1.9 \\
\hline 1988 & 493 & 1.5 & 165 & 0.5 & 658 & 2.0 \\
\hline 1989 & 555 & 1.7 & 165 & 0.5 & 720 & 2.2 \\
\hline 1990 & 597 & 1.8 & 165 & 0.5 & 762 & 2.3 \\
\hline 1991 & 650 & 1.9 & 165 & 0.5 & 815 & 2.4 \\
\hline $\begin{array}{l}\text { Nine-year } \\
\text { total }\end{array}$ & 4,176 & 12.6 & 1,485 & 4.5 & 5,661 & 17.1 \\
\hline
\end{tabular}

Note: This projection is based on a stock figure of 32,950 full-time university teachers in 1980-81 and assumes zero net mobility. The mortality rate is an approximation. The stock figure is held constant for the projection period, an assumption that must be qualified if universities, for financial and other reasons, reduce the total number of full-time faculty over the next nine years. 


\begin{tabular}{|c|c|c|c|c|c|c|c|}
\hline Field of Study & $\begin{array}{c}\text { Full-time and } \\
\text { part-time } \\
\text { doctoral } \\
\text { students } \\
1982-83^{p}\end{array}$ & $\begin{array}{l}\text { Foreign } \\
\text { students }\end{array}$ & $\begin{array}{l}\text { Less } \\
\text { fore1gn } \\
\text { students }\end{array}$ & $\begin{array}{l}\text { Withdrawal } \\
\text { rate in } \\
\text { percentage }\end{array}$ & Balance & $\begin{array}{l}\text { Length of } \\
\text { study 1n } \\
\text { years }\end{array}$ & $\begin{array}{c}\text { Degrees } \\
\text { granted } \\
\text { each year } \\
1983 \text { to } 1987\end{array}$ \\
\hline Education & 1,731 & 194 & 1,537 & 55 & 692 & 173 & 192 \\
\hline $\begin{array}{l}\text { Music } \\
F \perp \text { ne and Performing Arts }\end{array}$ & $\begin{array}{l}78 \\
48\end{array}$ & $\begin{array}{l}5 \\
8\end{array}$ & $\begin{array}{l}73 \\
40\end{array}$ & $\begin{array}{l}50 \\
50\end{array}$ & $\begin{array}{l}37 \\
20\end{array}$ & $\begin{array}{l}5 \\
5\end{array}$ & $\begin{array}{l}7 \\
4\end{array}$ \\
\hline SUB-TOTAL: FINE AND APPLIED ARTS & 126 & 13 & 113 & 50 & 57 & 5 & 11 \\
\hline Classics & 52 & 12 & 40 & 30 & 28 & 4 & 7 \\
\hline English & 595 & 106 & 489 & 50 & 245 & 4 & 61 \\
\hline French & 218 & 28 & 190 & 50 & 95 & 4 & 24 \\
\hline Other Languages & 323 & 68 & 255 & 50 & 128 & 5 & 26 \\
\hline History & 444 & 47 & 397 & 45 & 218 & 4 & 54 \\
\hline Library Science & 22 & 2 & 20 & 50 & 10 & 4 & 3 \\
\hline Linguistics & 164 & 29 & 135 & 50 & 68 & 4 & 17 \\
\hline Media Studies & 22 & 3 & 19 & 50 & 10 & 4 & 2 \\
\hline Ph1losophy & 478 & 95 & 383 & 50 & 192 & 5 & 38 \\
\hline Religious Studies & 270 & 49 & 221 & 50 & 111 & 5 & 22 \\
\hline SUB-TOTAL: HUMANITIES & 2,588 & 439 & 2,149 & 30 to 50 & 1,105 & 4 & 254 \\
\hline Anthropology and Archaeology & 272 & 42 & 230 & 50 & 115 & 4 & 29 \\
\hline Management & 213 & 33 & 180 & 50 & 90 & 4 & 22 \\
\hline Area Studies & 174 & 79 & 95 & 50 & 48 & 4 & 12 \\
\hline Economics & 496 & 204 & 292 & 50 & 146 & 4 & 36 \\
\hline Geography & 232 & 74 & 158 & 40 & 95 & 4 & 24 \\
\hline Law & 67 & 18 & 49 & 50 & 25 & 4 & 6 \\
\hline Man and Environmental Studies & 39 & 7 & 32 & 55 & 14 & 4 & 4 \\
\hline Polftical Sclence & 431 & 111 & 320 & 50 & 160 & 4 & 40 \\
\hline Psychology & 1,210 & 138 & 1,072 & 50 & 536 & 4 & 134 \\
\hline Soctal Work & 64 & 3 & 61 & 50 & 30 & 4 & 8 \\
\hline Sociology & 513 & 100 & 413 & 50 & 206 & 4 & 52 \\
\hline SUB-TOTAL: SOCIAL SCIENCES & 3,711 & 809 & 2,902 & 50 & 1,465 & 4 & 367 \\
\hline TOTAL HUMAN SCIENCES & 8,156 & 1,455 & 6,701 & 40 to 55 & 3,319 & 4 to 5 & 805 \\
\hline
\end{tabular}


Hounghold sctence

Household Sclence

Veterinary Science

Zoology

SUB-TOTAL: AGRICULTURE AND BIOLOGICAL SCIENCES 1,34

Chemical Engineering

Civil Engineering

Electrical Engineering

Mechantcal Engineering

Other Engineering

SUB-TOTAL: ENGINEERING

Forestry

TOTAL: ENGINEERING AND APPLIED SCIENCES

$\begin{array}{rr}109 & 231 \\ 33 & 107 \\ 110 & 39 \\ 19 & 54 \\ 5 & 24 \\ 7 & 29 \\ 37 & 187 \\ 320 & 1,024\end{array}$

231
107
392
54
24
29
187
1,024

25
25
40
40
50
50
40
40
25 to 50

\section{Dentistry}

Basic Medical Sciences

Pharmacy

Other Health Sciences

SUB-TOTAL: HEALTH SCIENCES

Computer Science

Mathematics

Chemistry

Geology

Other Physical Sciences

SUB-TOTAL: MATHEMATICS AND PHYSICAL SCIENCES

$$
\begin{array}{r}
496 \\
991
\end{array}
$$

TOTAL SCIENCES

Note: The rationale and methodology for this simulation exercise has been outlined by the author in the following publications: "The Ph.D. Dilemma in Canada: A Case Study", Canadian Higher Education in the Seventies, Economic Counc1l of Canada, May 1972, pp. 75-131; "The Ph.D. Dilemma in Canada Revisited", The Canadian Journal of Higher Education, February 1978, Pp. 49-92. 
As already indicated, in most disciplines, the number of retirements over the next five years will be small: 1,881 full-time faculty members will reach the normal retirement age of 65 between 1983 and 1987. This figure is much lower than the anticipated supply of Ph.D. graduates during the same five years - well over 7,000 (Table 8). Furthermore, the chances of new graduates securing a university teaching position may be even slimmer than these numbers suggest, as not everyone who reaches 65 retires. It should also be remembered that all university appointments do not necessarily go to Ph.D.-holders; in some cases, foreign professors and persons from other employment sectors will be hired as well as returning Canadians with foreign earned doctorates.

\section{CONCLUDING OBSERVATIONS}

Changes in enrolment patterns, age structure, rank distribution and salary level of full-time faculty are affecting Canadian universities. This coincides with a severe curtailment of government spending.

Enrolment in recent years has grown more rapidly than full-time faculty, resulting in an increase in the student/teacher ratio, and a possible decline in the quality of university education and research. High student/teacher ratios are particularly evident in professional disciplines. For example, the ratio in business was 31.7 to one, 18.7 in engineering, and 18.3 in law, compared with 14.2 for all disciplines.

Slow growth of full-time faculty has meant a decrease in employment opportunities for recent $\mathrm{Ph} . \mathrm{D}$. graduates (especially in the arts and sciences) and a general aging of the faculty. The impact of this aging process on university teaching and research needs to be assessed. A further consequence of the hiring cutback has been a substantial decline in mobility and fewer foreign faculty. It is anticipated that these trends will persist in the next few years.

The median salary of university professors has risen more rapidly than the Consumer Price Index (CPI), and is likely to continue to do so. Salary structure reflects rank distribution. In $1982-83,73 \%$ of the full-time university teachers will be either full or associate professors. Within a few years, four out of five may be in this senior category.

Under the best of circumstances, for the next few years, the annual replacement demand for full-time university teachers will be at around 500, whereas the supply of new doctoral degree-holders alone will be two or three times as high. This potential imbalance remains a cause for concern. 